\title{
EXTENDING THE EXTENSIONAL LAMBDA CALCULUS WITH SURJECTIVE PAIRING IS CONSERVATIVE
}

\author{
KRISTIAN STØVRING
}

BRICS, Department of Computer Science, University of Aarhus, IT-parken, Aabogade 34, DK-8200

Aarhus N, Denmark

e-mail address: kss@brics.dk

ABstract. We answer Klop and de Vrijer's question whether adding surjective-pairing axioms to the extensional lambda calculus yields a conservative extension. The answer is positive. As a byproduct we obtain a "syntactic" proof that the extensional lambda calculus with surjective pairing is consistent.

\section{INTRODUCTION}

The theory $\lambda_{\beta \eta \mathrm{SP}}$ is obtained from the untyped extensional lambda calculus $\lambda_{\beta \eta}$ [2] by adding three surjective-pairing axioms:

$$
\begin{array}{rrrl}
\left(\pi_{1}\right) & \pi_{1}\langle M, N\rangle & =M \\
\left(\pi_{2}\right) & \pi_{2}\langle M, N\rangle & =N \\
\text { (sP) } & \left\langle\pi_{1} M, \pi_{2} M\right\rangle & =M
\end{array}
$$

These axioms are said to define a surjective pairing since the axiom (SP) implies that every term is equal to a pair.

A $\lambda$-term is called pure if it does not contain any of the new constructs $\pi_{i}$ and $\langle\cdot, \cdot\rangle$. In this article we give a positive answer to the following question, asked by Klop and de Vrijer in 1989 [10, 23] and featured as Problem 5 in the original RTA list of open problems [6]:

Suppose that $M$ and $N$ are pure $\lambda$-terms. Does $M={ }_{\beta \eta \mathrm{SP}} N$ imply that $M={ }_{\beta \eta} N ?$

In other words, we show that the theory $\lambda_{\beta \eta \mathrm{SP}}$ is a conservative extension of the theory $\lambda_{\beta \eta}$. As a byproduct we obtain a proof of consistency of $\lambda_{\beta \eta \text { SP }}$ that uses purely syntactic methods. $^{1}$

2000 ACM Subject Classification: F.4.1.

Key words and phrases: Lambda calculus, surjective pairing, extensionality.

$1^{1}$ The author only knows of one other such "syntactic" consistency proof for $\lambda_{\beta \eta \mathrm{SP}}$, namely one based on recent work on operationally defined bisimulations 12 .

\section{LOGICAL METHODS} IN COMPUTER SCIENCE
DOI:10.2168/LMCS-2 (2:1) 2006

(C) K. Støvring (c) Creative Commons 
1.1. Background of the problem. The two perhaps most obvious attempts at showing conservativity of $\lambda_{\beta \eta \mathrm{SP}}$ fail because of two negative results: no surjective-pairing function (that is, no pairing function satisfying the three axioms above) is definable in the lambda calculus [1], and the standard reduction relation for the lambda calculus with surjective pairing is not confluent [9]. Both results were shown for the extensional lambda calculus as well.

Klop [9] and Klop and de Vrijer [10] have considered a number of properties of the (non-extensional) lambda calculus with surjective pairing, $\lambda_{\beta S P}$, which would have trivially followed from confluence of the standard reduction relation. In particular, de Vrijer has shown that $\lambda_{\beta S P}$ is a conservative extension of the lambda calculus [23. This result motivated the question answered here: whether surjective pairing also conservatively extends the extensional lambda calculus.

The proof of conservativity by de Vrijer is furthermore the first known "syntactic" consistency proof for $\lambda_{\beta S P}$. One of Scott's model-theoretic consistency proofs for $\lambda_{\beta \eta}[20$ ] can be easily adapted to show consistency of $\lambda_{\beta \eta \mathrm{SP}}$ (and hence also $\lambda_{\beta \mathrm{SP}}$ ) as well.

The theory $\lambda_{\beta \eta \text { SP }}$ has also been investigated from a categorical point of view. If $\mathcal{C}$ is a cartesian closed category with an object $U$ such that

$$
U \cong U \times U \cong U \rightarrow U
$$

then there are various ways of interpreting $\lambda$-terms as morphisms of $\mathcal{C}$ [2, 11]. Moreover, every extension of the theory $\lambda_{\beta \eta \mathrm{SP}}$ is the theory of a model arising in this way [11, 21].

1.2. Formalization. The author has formalized and verified the proof of the conservativity result using the Twelf system [16]. The formalized proof additionally serves as an implementation of a procedure transforming a formal derivation of $M={ }_{\beta \eta \mathrm{SP}} N$ into a formal derivation of $M={ }_{\beta \eta} N$ (for pure terms $M$ and $N$ ). It is available from

$$
\text { http://purl.oclc.org/net/kss/eta-SP }
$$

The formalized statement of the main result is presented in Appendix A.

\section{BACKGROUND AND NOTATION}

The reader is assumed to be familiar with basic properties of the untyped lambda calculus, as presented for example in the first three chapters of Barendregt's book [2].

The syntax of $\lambda$-terms is extended with constructs for pairing and projection:

$$
M::=x|\lambda x . M| M M|\langle M, M\rangle| \pi_{1} M \mid \pi_{2} M
$$

(where $x$ ranges over an infinite set of variables). The pure terms are the usual $\lambda$-terms, i.e., terms with no occurrences of $\pi_{i}$ or $\langle\cdot, \cdot\rangle$. The set of free variables of a term $M$ is denoted $\mathrm{FV}(M)$. We follow practice and identify $\alpha$-equivalent terms.

We use the following notation and definitions for relations on $\lambda$-terms: For any binary relation $\triangleright_{\mathcal{R}}$ on $\lambda$-terms, $\longrightarrow \mathcal{R}$ denotes the compatible closure of $\triangleright_{\mathcal{R}}$ as defined in Figure 1. The relation $\longrightarrow_{\mathcal{R}}$ is called a reduction relation. The reflexive-transitive closure of $\longrightarrow \mathcal{R}$

is written $\longrightarrow_{\mathcal{R}}^{*}$, and the reflexive-transitive-symmetric closure of $\longrightarrow_{\mathcal{R}}$ is written $=_{\mathcal{R}}$. We write $\lambda_{\mathcal{R}}$ for the equational theory of $\lambda$-terms corresponding to $=_{\mathcal{R}}$, i.e., $\lambda_{\mathcal{R}}$ is the set of formal equations " $M=N$ " such that $M={ }_{\mathcal{R}} N$.

The relation $\triangleright_{\beta \eta S P}$ is defined by the axioms in Figure 2. This relation generates a reduction relation $\longrightarrow \beta \eta$ SP and an equality relation $=\beta \eta \mathrm{SP}$. The extensional lambda calculus with surjective pairing is defined as the theory $\lambda_{\beta \eta \mathrm{SP}}$. 


$$
\begin{array}{cc}
\frac{M \triangleright_{\mathcal{R}} M^{\prime}}{M \longrightarrow_{\mathcal{R}} M^{\prime}} & \frac{M \longrightarrow_{\mathcal{R}} M^{\prime}}{\lambda x . M \longrightarrow_{\mathcal{R}} \lambda x \cdot M^{\prime}} \\
\frac{M \longrightarrow_{\mathcal{R}} M^{\prime}}{M N \longrightarrow_{\mathcal{R}} M^{\prime} N} & \frac{N \longrightarrow_{\mathcal{R}} N^{\prime}}{M N \longrightarrow_{\mathcal{R}} M^{\prime}} \\
\frac{M \longrightarrow_{\mathcal{R}} M^{\prime}}{\langle M, N\rangle \longrightarrow_{\mathcal{R}}\left\langle M^{\prime}, N\right\rangle} & \frac{N \longrightarrow_{\mathcal{R}} N^{\prime}}{\langle M, N\rangle \longrightarrow_{\mathcal{R}}\left\langle M, N^{\prime}\right\rangle} \\
\frac{M \longrightarrow_{\mathcal{R}} M^{\prime}}{\pi_{1} M \longrightarrow_{\mathcal{R}} \pi_{1} M^{\prime}} & \frac{M \longrightarrow_{\mathcal{R}} M^{\prime}}{\pi_{2} M{ }_{\mathcal{R}} \pi_{2} M^{\prime}}
\end{array}
$$

Figure 1: The compatible closure of $\triangleright_{\mathcal{R}}$.

$\begin{array}{lrlll}(\beta) & (\lambda x . M) N & \triangleright_{\beta \eta \mathrm{SP}} & M[x:=N] & \\ (\eta) & \lambda x . M x & \triangleright_{\beta \eta \mathrm{SP}} & M & \text { (if } x \notin \mathrm{FV}(M)) \\ \left(\pi_{1}\right) & \pi_{1}\langle M, N\rangle & \triangleright_{\beta \eta \mathrm{SP}} & M & \\ \left(\pi_{2}\right) & \pi_{2}\langle M, N\rangle & \triangleright_{\beta \eta \mathrm{SP}} & N & \\ (\mathrm{SP}) & \left\langle\pi_{1} M, \pi_{2} M\right\rangle & \triangleright_{\beta \eta \mathrm{SP}} & M & \end{array}$

Figure 2: The relation $\triangleright_{\beta \eta \mathrm{SP}}$.

\section{Overview OF THE PROOF}

The relation $\longrightarrow \beta \eta \mathrm{SP}$ is the standard reduction relation generating $=_{\beta \eta \mathrm{SP}}$. This reduction relation is, however, not confluent [5] 9, p. 216]; its confluence would immediately imply the main result, namely that $\lambda_{\beta \eta \mathrm{SP}}$ is conservative over $\lambda_{\beta \eta}{ }^{2}$

In this article we instead consider a further extension $\lambda_{\mathrm{FP}}$ of $\lambda_{\beta \eta \mathrm{SP}}$ and show that $\lambda_{\mathrm{FP}}$ is conservative over $\lambda_{\beta \eta}$. Since $\lambda_{\mathrm{FP}}$ is an extension of $\lambda_{\beta \eta \mathrm{SP}}$, the main result follows. The proof is structured in the following way:

- In Section 4 we present the extension $\lambda_{\mathrm{FP}}$ of $\lambda_{\beta \eta \mathrm{SP}}$ and show that it is generated by a confluent reduction relation $\longrightarrow$ FP. In the relation $\longrightarrow$ FP the orientation of the axioms $(\eta)$ and (SP) is reversed; in other words, the extensionality axioms are oriented as expansion axioms (see, e.g., the work by Jay and Ghani [8]).

- In Section 5 we show that $\lambda_{\mathrm{FP}}$ is conservative over $\lambda_{\beta \eta}$ on pure $\lambda$-terms. This result does not immediately follow from confluence of $\longrightarrow$ FP since $\longrightarrow$ FP contains (SP) oriented as an expansion axiom.

\footnotetext{
${ }^{2}$ The non-confluent reduction relation considered by Klop [9] is slightly different from $\longrightarrow \beta \eta \mathrm{SP}$. It is simple to construct a counter-example to confluence similar to Klop's.
} 


\section{An extension of the theory $\lambda_{\beta \eta S P}$}

We first present the extension $\lambda_{\mathrm{FP}}$ of $\lambda_{\beta \eta \mathrm{SP}}$; the name FP is intended to be a mnemonic for "functional pairing". The relation $\triangleright_{\mathrm{FP}}$ is defined by the axioms in Figure 3. This relation generates the theory $\lambda_{\mathrm{FP}}$ and the reduction relation $\longrightarrow$ FP. For convenience, we refer to the axioms $(\delta \pi),\left(\pi_{1} \lambda\right)$, and $\left(\pi_{2} \lambda\right)$ as the commutation axioms; intuitively, these axioms express how a function behaves as a pair and vice versa. As discussed above, the axioms $(\bar{\eta})$ and $(\overline{\mathrm{SP}})$ are oriented as expansion axioms.

The theory $\lambda_{\mathrm{FP}}$ it not new, although is does not appear to have been explicitly named before. Axioms similar to the commutation axioms $(\delta \pi),\left(\pi_{1} \lambda\right)$, and $\left(\pi_{2} \lambda\right)$ were first considered in work on products and lists in the lambda calculus [18 and in work on categorical combinators [19]: adding the surjective-pairing axiom (SP) to Révész's theory $\lambda_{p}$ gives the theory $\lambda_{\mathrm{FP}}$, except for a minor syntactic difference. Durfee gave a model for the full theory $\lambda_{\mathrm{FP}}$ [7] (see the remark below). Axioms equivalent to the commutation axioms play an indirect, but important, role in recent work on solvability for $\lambda$-terms with pairs [12].

The reduction relation $\longrightarrow$ FP (with its combination of commutation axioms and expansion axioms) appears to be new.

$\begin{array}{rrrll}(\beta) & (\lambda x . M) N & \triangleright_{\mathrm{FP}} & M[x:=N] & \\ (\bar{\eta}) & M & \triangleright_{\mathrm{FP}} & \lambda x . M x & (\text { if } x \notin \mathrm{FV}(M)) \\ \left(\pi_{1}\right) & \pi_{1}\langle M, N\rangle & \triangleright_{\mathrm{FP}} & M & \\ \left(\pi_{2}\right) & \pi_{2}\langle M, N\rangle & \triangleright_{\mathrm{FP}} & N \\ (\overline{\mathrm{SP}}) & M & \triangleright_{\mathrm{FP}} & \left\langle\pi_{1} M, \pi_{2} M\right\rangle & \\ (\delta \pi) & \langle M, N\rangle P & \triangleright_{\mathrm{FP}} & \langle M P, N P\rangle & \\ \left(\pi_{1} \lambda\right) & \pi_{1}(\lambda x . M) & \triangleright_{\mathrm{FP}} & \lambda x \cdot \pi_{1} M \\ \left(\pi_{2} \lambda\right) & \pi_{2}(\lambda x . M) & \triangleright_{\mathrm{FP}} & \lambda x \cdot \pi_{2} M & \end{array}$

Figure 3: The relation $\triangleright_{\mathrm{FP}}$.

Remark. In this article, the theory $\lambda_{\mathrm{FP}}$ and the associated reduction relation $\longrightarrow_{\mathrm{FP}}$ are used to prove a specific result about a different theory. However, $\lambda_{\mathrm{FP}}$ and $\longrightarrow_{\mathrm{FP}}$ can be justified semantically and syntactically:

- From the point of view of semantics: The original model of $\lambda_{\beta \eta S P}$ [11, 20] is also a model of $\lambda_{\mathrm{FP}}$ 7]. Indeed, let $U$ and $V$ be complete partial orders such that $V \cong V \times V$ and $U \cong[U \rightarrow V]$. Then by calculations valid in any cartesian closed category [20, $U \cong U \times U \cong[U \rightarrow U]$, and one can verify that the standard interpretation $^{3}$ of $\lambda$-terms as elements of $U$ gives rise to a model of $\lambda_{\mathrm{FP}}$.

As an aside, if $U$ is an arbitrary complete partial order satisfying that $U \cong$ $U \times U \cong[U \rightarrow U]$, then the standard interpretation using these isomorphisms makes $U$ a model of (at least) $\lambda_{\beta \eta \mathrm{SP}}$. Taking $U=V$ in the above construction now gives an alternative pair of isomorphisms, and hence an alternative interpretation of $\lambda$-terms, resulting in a model of $\lambda_{\mathrm{FP}}$.

\footnotetext{
${ }^{3}$ See also Exercise 18.4.19 in Barendregt's book [2].
} 
- From the point of view of term rewriting: In the simply-typed lambda calculus, term constructs can be proof-theoretically classified as either introduction forms $(\lambda x . M$ and $\langle M, N\rangle)$ or elimination forms $\left(M N\right.$ and $\left.\pi_{i} M\right)$, using the Curry-Howard isomorphism [3. The simply-typed counterparts of the axioms $(\beta),\left(\pi_{1}\right)$, and $\left(\pi_{2}\right)$ of Figure 3 then imply that, when constructing a term bottom-up, "an introduction form followed by an elimination form is a redex." This property is preserved in the untyped reduction relation $\longrightarrow$ FP by virtue of the commutation axioms $(\delta \pi),\left(\pi_{1} \lambda\right)$ and $\left(\pi_{2} \lambda\right)$.

In the rest of this section we prove that $\longrightarrow_{F P}$ is confluent. For that purpose we describe $\longrightarrow_{\text {FP }}$ as the union of two relations: a part $\longrightarrow$ E generated from the $\eta$ /sP-expansion axioms $(\bar{\eta})$ and $(\overline{\mathrm{SP}})$, and an "extensionality-free" part $\longrightarrow_{\mathrm{R}}$ generated from all the remaining axioms.

- In Section 4.1 we show that the extensionality-free part $\longrightarrow_{\mathrm{R}}$ is confluent.

- In Section 4.2 we review the well-known fact that $\eta /$ SP-expansion $\longrightarrow_{\mathrm{E}}$ is confluent, and then show that $\longrightarrow_{\mathrm{E}}$ commutes with $\longrightarrow_{\mathrm{R}}$ : if $N_{1} \longleftarrow_{\mathrm{E}}^{*} M \longrightarrow_{\mathrm{R}}^{*} N_{2}$, then there is a $P$ such that $N_{1} \longrightarrow{ }_{\mathrm{R}}^{*} P \longleftarrow{ }_{\mathrm{E}}^{*} N_{2}$.

We conclude by the Hindley-Rosen Lemma [2, p. 64] that the union $\longrightarrow_{\mathrm{FP}}=\longrightarrow_{\mathrm{R}} \cup \longrightarrow_{\mathrm{E}}$ is confluent. Earlier, van Oostrom used a similar approach to prove confluence of $\eta$-expansion (together with $\beta$-reduction) in the pure lambda calculus [14.

From a technical point of view, the proof that $\longrightarrow_{\mathrm{E}}$ commutes with $\longrightarrow_{\mathrm{R}}$ is the novel part of the confluence proof: the commutation proof highlights the role of the axioms $(\delta \pi)$, $\left(\pi_{1} \lambda\right)$, and $\left(\pi_{2} \lambda\right)$.

4.1. Confluence of an extensionality-free subrelation. The relation $\triangleright_{\mathrm{R}}$ is defined by all the axioms of $\triangleright_{\mathrm{FP}}$ except $(\bar{\eta})$ and $(\overline{\mathrm{SP}})$; for convenience the remaining axioms are shown in Figure 4. The relation $\triangleright_{\mathrm{R}}$ generates the reduction relation $\longrightarrow_{\mathrm{R}}$.

$\begin{array}{cccl}(\beta) & (\lambda x . M) N & \triangleright_{\mathrm{R}} & M[x:=N] \\ \left(\pi_{1}\right) & \pi_{1}\langle M, N\rangle & \triangleright_{\mathrm{R}} & M \\ \left(\pi_{2}\right) & \pi_{2}\langle M, N\rangle & \triangleright_{\mathrm{R}} & N \\ (\delta \pi) & \langle M, N\rangle P & \triangleright_{\mathrm{R}} & \langle M P, N P\rangle \\ \left(\pi_{1} \lambda\right) & \pi_{1}(\lambda x . M) & \triangleright_{\mathrm{R}} & \lambda x \cdot \pi_{1} M \\ \left(\pi_{2} \lambda\right) & \pi_{2}(\lambda x . M) & \triangleright_{\mathrm{R}} & \lambda x \cdot \pi_{2} M\end{array}$

Figure 4: The relation $\triangleright_{\mathrm{R}}$.

We now aim to prove that $\longrightarrow_{R}$ is confluent. In fact, this follows from general higherorder rewriting theory, since $\longrightarrow \mathrm{R}$ can be formulated as an orthogonal pattern higher-order rewriting system [13, 17, and such systems are confluent [13. However, in order to keep the presentation self-contained, we give a direct confluence proof. This direct proof, which follows the method of the Tait/Martin-Löf proof of confluence of $\beta$-reduction [2, p. 60], can be viewed as a specialized version of Nipkow's confluence proof [13]. 
First, define a parallel [22] reduction relation $\Longrightarrow_{\mathrm{R}}$, shown in Figure ${ }^{4}$

$$
\begin{aligned}
& \frac{M \Longrightarrow_{\mathrm{R}} M^{\prime} \quad N \Longrightarrow_{\mathrm{R}} N^{\prime}}{(\lambda x \cdot M) N \Longrightarrow_{\mathrm{R}} M^{\prime}\left[x:=N^{\prime}\right]} \\
& \frac{M \Longrightarrow_{\mathrm{R}} M^{\prime}}{\pi_{1}\langle M, N\rangle \Longrightarrow_{\mathrm{R}} M^{\prime}} \quad \frac{N \Longrightarrow_{\mathrm{R}} N^{\prime}}{\pi_{2}\langle M, N\rangle \Longrightarrow_{\mathrm{R}} N^{\prime}} \\
& \frac{M \Longrightarrow_{\mathrm{R}} M^{\prime} \quad N \Longrightarrow_{\mathrm{R}} N^{\prime} \quad P \Longrightarrow_{\mathrm{R}} P^{\prime}}{\langle M, N\rangle P \Longrightarrow M_{\mathrm{R}}\left\langle M^{\prime} P^{\prime}, N^{\prime} P^{\prime}\right\rangle} \\
& \frac{M \Longrightarrow_{\mathrm{R}} M^{\prime}}{\pi_{1}(\lambda x . M) \Longrightarrow_{\mathrm{R}} \lambda x \cdot \pi_{1} M^{\prime}} \quad \frac{M \Longrightarrow_{\mathrm{R}} M^{\prime}}{\pi_{2}(\lambda x \cdot M) \Longrightarrow_{\mathrm{R}} \lambda x \cdot \pi_{2} M^{\prime}} \\
& \overline{M \Longrightarrow_{\mathrm{R}} M} \quad \frac{M \Longrightarrow_{\mathrm{R}} M^{\prime}}{\lambda x \cdot M \Longrightarrow_{\mathrm{R}} \lambda x \cdot M^{\prime}} \\
& \frac{M \Longrightarrow_{\mathrm{R}} M^{\prime} N \Longrightarrow_{\mathrm{R}} N^{\prime}}{M N \Longrightarrow_{\mathrm{R}} M^{\prime} N^{\prime}} \quad \frac{M \Longrightarrow_{\mathrm{R}} M^{\prime} N \Longrightarrow_{\mathrm{R}} N^{\prime}}{\langle M, N\rangle \Longrightarrow_{\mathrm{R}}\left\langle M^{\prime}, N^{\prime}\right\rangle} \\
& \frac{M \Longrightarrow_{\mathrm{R}} M^{\prime}}{\pi_{1} M \Longrightarrow_{\mathrm{R}} \pi_{1} M^{\prime}} \quad \frac{M \Longrightarrow_{\mathrm{R}} M^{\prime}}{\pi_{2} M \Longrightarrow_{\mathrm{R}} \pi_{2} M^{\prime}}
\end{aligned}
$$

Figure 5: Parallel R-reduction $\Longrightarrow_{\mathrm{R}}$.

\section{Proposition 4.1.}

(i) $\longrightarrow_{\mathrm{R}}^{*}=\Longrightarrow_{\mathrm{R}}^{*}$.

(ii) If $M \Longrightarrow_{\mathrm{R}} M^{\prime}$ and $N \Longrightarrow_{\mathrm{R}} N^{\prime}$, then $M[x:=N] \Longrightarrow_{\mathrm{R}} M^{\prime}\left[x:=N^{\prime}\right]$.

(iii) If $M \longrightarrow{ }_{\mathrm{R}}^{*} M^{\prime}$ and $N \longrightarrow{ }_{\mathrm{R}}^{*} N^{\prime}$, then $M[x:=N] \longrightarrow{ }_{\mathrm{R}}^{*} M^{\prime}\left[x:=N^{\prime}\right]$.

Proof. Standard [2, p. 60]. Part (iii) follows from the first two parts and will be used in the next section.

Proposition 4.2. The relation $\Longrightarrow_{\mathrm{R}}$ satisfies the diamond property: if $M \Longrightarrow_{\mathrm{R}} N_{1}$ and $M \Longrightarrow_{\mathrm{R}} N_{2}$, then there is a $P$ such that $N_{1} \Longrightarrow_{\mathrm{R}} P$ and $N_{2} \Longrightarrow_{\mathrm{R}} P$.

Proof. By induction on the derivations of $M \Longrightarrow_{\mathrm{R}} N_{1}$ and $M \Longrightarrow_{\mathrm{R}} N_{2}$ according to the rules in Figure 5 . Many of the cases are well-known from the proof of confluence of $\beta$-reduction. There are no interesting new cases (which is another way of saying that $\longrightarrow_{R}$ can naturally be defined as an orthogonal higher-order term rewriting system).

\footnotetext{
${ }^{4}$ The notion that $\Longrightarrow_{R}$ is the parallel reduction relation generated from the axioms of $\triangleright_{\mathrm{R}}$ can be made precise [13] Section 4].
} 
Corollary 4.3. The relation $\longrightarrow_{\mathrm{R}}$ is confluent.

4.2. The relation $\longrightarrow_{\mathrm{R}}$ commutes with $\eta$ /SP-expansion. We define the relation $\triangleright_{\mathrm{E}}$ by the axioms $(\bar{\eta})$ and $(\overline{\mathrm{SP}})$, for convenience shown in Figure 6 . This relation generates the $\eta$ /SP-expansion relation $\longrightarrow \mathrm{E}$.

$$
\begin{array}{lllll}
(\bar{\eta}) & M & \triangleright_{\mathrm{E}} & \lambda x \cdot M x & (\text { if } x \notin \mathrm{FV}(M)) \\
(\overline{\mathrm{SP}}) & M & \triangleright_{\mathrm{E}} & \left\langle\pi_{1} M, \pi_{2} M\right\rangle &
\end{array}
$$

Figure 6: The relation $\triangleright_{\mathrm{E}}$.

The purpose of this section is to show that $\longrightarrow_{\mathrm{E}}$ commutes with $\longrightarrow_{\mathrm{R}}$, that is, if

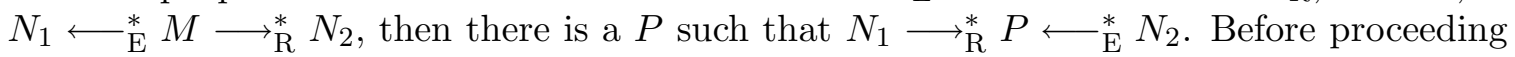
with the proof of commutation, we consider some of the critical pairs [13] between $\longrightarrow_{\mathrm{E}}$ and $\longrightarrow \mathrm{R}$. The first two cases are well-known:

(1) $(\lambda x .(\lambda x . M) x) N \longleftarrow \bar{\eta}(\lambda x . M) N \longrightarrow \beta M[x:=N]$.

Solution: $(\lambda x .(\lambda x . M) x) N \longrightarrow \beta(\lambda x . M) N \longrightarrow_{\beta} M[x:=N]$.

(2) $\pi_{i}\left\langle\pi_{1}\left\langle M_{1}, M_{2}\right\rangle, \pi_{2}\left\langle M_{1}, M_{2}\right\rangle\right\rangle \longleftarrow \overline{\mathrm{SP}} \pi_{i}\left\langle M_{1}, M_{2}\right\rangle \longrightarrow \pi_{i} M_{i}$.

Solution: $\pi_{i}\left\langle\pi_{1}\left\langle M_{1}, M_{2}\right\rangle, \pi_{2}\left\langle M_{1}, M_{2}\right\rangle\right\rangle \longrightarrow \pi_{i} \pi_{i}\left\langle M_{1}, M_{2}\right\rangle \longrightarrow \pi_{i} M_{i}$.

On the other hand, to resolve the next two kinds of critical pairs, one needs the commutation axioms $(\delta \pi),\left(\pi_{1} \lambda\right)$, and $\left(\pi_{2} \lambda\right)$ :

(3) $\pi_{1}\left(\lambda x .\left\langle M_{1}, M_{2}\right\rangle x\right) \longleftarrow_{\bar{\eta}} \pi_{i}\left\langle M_{1}, M_{2}\right\rangle \longrightarrow_{i} M_{i}$.

Solution:

$$
\begin{array}{rll}
\pi_{i}\left(\lambda x .\left\langle M_{1}, M_{2}\right\rangle x\right) & \longrightarrow \delta \pi & \pi_{i}\left(\lambda x \cdot\left\langle M_{1} x, M_{2} x\right\rangle\right) \\
& \longrightarrow \pi_{i} \lambda & \lambda x \cdot \pi_{i}\left\langle M_{1} x, M_{2} x\right\rangle \\
& \longrightarrow \pi_{i} & \lambda x \cdot M_{i} x \\
& \longleftarrow & M_{i} .
\end{array}
$$

(4) $\left\langle\pi_{1}(\lambda x . M), \pi_{2}(\lambda x \cdot M)\right\rangle N \longleftarrow \overline{\mathrm{SP}}(\lambda x \cdot M) N \longrightarrow_{\beta} M[x:=N]$.

Solution:

$$
\begin{array}{rll}
\left\langle\pi_{1}(\lambda x . M), \pi_{2}(\lambda x . M)\right\rangle N & \longrightarrow \pi_{1} \lambda, \pi_{2} \lambda & \left\langle\lambda x \cdot \pi_{1} M, \lambda x \cdot \pi_{2} M\right\rangle N \\
& \longrightarrow \delta \pi & \left\langle\left(\lambda x \cdot \pi_{1} M\right) N,\left(\lambda x \cdot \pi_{2} M\right) N\right\rangle \\
& \longrightarrow \beta & \left\langle\pi_{1}(M[x:=N]), \pi_{2}(M[x:=N])\right\rangle \\
& \longleftarrow & M[x:=N] .
\end{array}
$$

These are all the kinds of critical pairs between $\longrightarrow_{\mathrm{E}}$ and $\longrightarrow_{\mathrm{R}}$ in which the R-step uses one of the axioms $(\beta),\left(\pi_{1}\right)$, or $\left(\pi_{2}\right)$. The cases where the R-step is one of the remaining axioms can be resolved similarly to the simple cases 1 and 2 .

We now turn to the actual proof of commutation. Define a parallel $\eta /$ sP-expansion relation $\Longrightarrow_{\mathrm{E}}[8,22$ by the rules in Figure 7

First, some simple facts about parallel $\eta$ /sP-expansion:

\section{Proposition 4.4.}

(i) $\longrightarrow_{\mathrm{E}}^{*}=\mathrm{E}_{\mathrm{E}}^{*}$.

(ii) $\longrightarrow \mathrm{E}$ is confluent. 


$$
\begin{array}{cc}
\frac{M \Longrightarrow_{\mathrm{E}} M^{\prime}}{M \Longrightarrow_{\mathrm{E}} \lambda x \cdot M^{\prime} x}(x \notin \mathrm{FV}(M)) & \frac{M \Longrightarrow_{\mathrm{E}} M^{\prime}}{M \Longrightarrow_{\mathrm{E}}\left\langle\pi_{1} M^{\prime}, \pi_{2} M^{\prime}\right\rangle} \\
\frac{{ }_{\mathrm{E}} M}{M \Longrightarrow_{\mathrm{E}} M^{\prime} \quad N \Longrightarrow_{\mathrm{E}} N^{\prime}} & \frac{M \Longrightarrow_{\mathrm{E}} M^{\prime}}{\lambda x \cdot M \Longrightarrow_{\mathrm{E}} \lambda x \cdot M^{\prime}} \\
M \Longrightarrow_{\mathrm{E}} M^{\prime} N^{\prime} & M \Longrightarrow_{\mathrm{E}} M^{\prime} N \Longrightarrow_{\mathrm{E}} N^{\prime} \\
\frac{\langle M, N\rangle \Longrightarrow_{\mathrm{E}}\left\langle M^{\prime}, N^{\prime}\right\rangle}{\pi_{1} M \Longrightarrow_{\mathrm{E}} M^{\prime} \pi_{1} M^{\prime}} & \frac{M \Longrightarrow_{\mathrm{E}} M^{\prime}}{\pi_{2} M \Longrightarrow_{\mathrm{E}} \pi_{2} M^{\prime}}
\end{array}
$$

Figure 7: Parallel $\eta$ /sP-expansion $\Longrightarrow_{\mathrm{E}}$.

(iii) If $M \Longrightarrow_{\mathrm{E}} M^{\prime}$ and $N \Longrightarrow_{\mathrm{E}} N^{\prime}$, then $M[x:=N] \Longrightarrow_{\mathrm{E}} M^{\prime}\left[x:=N^{\prime}\right]$.

Proof. Standard [8]. The confluence of $\longrightarrow_{\mathrm{E}}$ follows from the diamond property of $\Longrightarrow_{\mathrm{E}}$.

We now aim to prove that if $N_{1} \Longleftarrow_{\mathrm{E}} M \longrightarrow_{\mathrm{R}} N_{2}$, then there exists a $P$ such that $N_{1} \longrightarrow_{\mathrm{R}}^{*} P \Longleftarrow{ }_{\mathrm{E}} N_{2}$. Consider for example the case

$$
N Q \Longleftarrow \mathrm{E}(\lambda x . M) Q \longrightarrow_{\beta} M[x:=Q]
$$

where $N \Longleftarrow \mathrm{E} \lambda x . M$. Then $N$ results from $\lambda x . M$ by a number of $\eta /$ sP-expansions, and in order to show commutation we intuitively need to iterate cases 1 and 4 of the critical pair calculations shown in the beginning of this section. Similar examples exist for the other axioms of $\longrightarrow_{\mathrm{R}}$. The properties which are needed are shown in the following two lemmas:

Lemma 4.5. If $\lambda x . M \Longrightarrow_{\mathrm{E}} N$, then

(i) there is a $P$ such that $N x \longrightarrow{ }_{\mathrm{R}}^{*} P \Longleftarrow_{\mathrm{E}} M$, and

(ii) there is a $Q$ such that for $i \in\{1,2\}: \pi_{i} N \longrightarrow_{\mathrm{R}}^{*} \lambda x \cdot \pi_{i} Q$ and $M \Longrightarrow_{\mathrm{E}} Q$.

Proof. By induction on the definition of $\lambda x . M \Longrightarrow_{\mathrm{E}} N$.

Lemma 4.6. If $\left\langle M_{1}, M_{2}\right\rangle \Longrightarrow_{\mathrm{E}} N$, then

(i) for $i \in\{1,2\}$ there is a $P_{i}$ such that $\pi_{i} N \longrightarrow{ }_{\mathrm{R}}^{*} P_{i} \Longleftarrow_{\mathrm{E}} M_{i}$, and

(ii) there are $Q_{1}, Q_{2}$ such that $N x \longrightarrow_{\mathrm{R}}^{*}\left\langle Q_{1} x, Q_{2} x\right\rangle$ and $M_{1} \Longrightarrow_{\mathrm{E}} Q_{1}$ and $M_{2} \Longrightarrow_{\mathrm{E}} Q_{2}$.

Proof. By induction on the definition of $\left\langle M_{1}, M_{2}\right\rangle \Longrightarrow_{\mathrm{E}} N$.

We now prove the main lemma needed in the commutation proof:

Lemma 4.7. If $N \Longleftarrow_{\mathrm{E}} M \longrightarrow_{\mathrm{R}} M^{\prime}$, then there is a $P$ such that $N \longrightarrow_{\mathrm{R}}^{*} P \Longleftarrow{ }_{\mathrm{E}} M^{\prime}$.

Proof. Induction on the definition of $M \Longrightarrow_{\mathrm{E}} N$, using Lemmas 4.5 and 4.6. We show some illustrative cases. 
Case 1: $\left\langle\pi_{1} N^{\prime}, \pi_{2} N^{\prime}\right\rangle \Longleftarrow_{\mathrm{E}} M \longrightarrow_{\mathrm{R}} M^{\prime}$ where $N^{\prime} \Longleftarrow_{\mathrm{E}} M$. By the induction hypothesis there is a $P^{\prime}$ such that $N^{\prime} \longrightarrow{ }_{\mathrm{R}}^{*} P^{\prime} \Longleftarrow_{\mathrm{E}} M^{\prime}$. Then

$$
\left\langle\pi_{1} N^{\prime}, \pi_{2} N^{\prime}\right\rangle \longrightarrow_{\mathrm{R}}^{*}\left\langle\pi_{1} P^{\prime}, \pi_{2} P^{\prime}\right\rangle \Longleftarrow_{\mathrm{E}} M^{\prime}
$$

so choose $P=\left\langle\pi_{1} P^{\prime}, \pi_{2} P^{\prime}\right\rangle$.

Case 2: $N_{1} N_{2} \Longleftarrow_{\mathrm{E}}\left(\lambda x \cdot M_{1}\right) M_{2} \longrightarrow_{\mathrm{R}} M_{1}\left[x:=M_{2}\right]$ where $N_{1} \Longleftarrow_{\mathrm{E}} \lambda x \cdot M_{1}$ and where $N_{2} \Longleftarrow_{\mathrm{E}} M_{2}$. By Lemma 4.5)(i) there is a $P^{\prime}$ such that $N_{1} x \longrightarrow_{\mathrm{R}}^{*} P^{\prime} \Longleftarrow_{\mathrm{E}} M_{1}$. It is easy to see from the definition of $\Longrightarrow_{\mathrm{E}}$ that $x$ is not free in $N_{1}$. Therefore, by Propositions 4.1 and $4.4 N_{1} N_{2} \longrightarrow_{\mathrm{R}}^{*} P^{\prime}\left[x:=N_{2}\right] \Longleftarrow_{\mathrm{E}} M_{1}\left[x:=M_{2}\right]$, so choose $P=P^{\prime}\left[x:=N_{2}\right]$.

\section{Lemma 4.8.}

(i) If $N{ }_{\mathrm{E}} M \longrightarrow{ }_{\mathrm{R}}^{*} M^{\prime}$, then there is a $P$ such that $N \longrightarrow_{\mathrm{R}}^{*} P{ }_{\mathrm{E}} M^{\prime}$.

(ii) If $N \Longleftarrow{ }_{\mathrm{E}}^{*} M \longrightarrow{ }_{\mathrm{R}}^{*} M^{\prime}$, then there is a $P$ such that $N \longrightarrow{ }_{\mathrm{R}}^{*} P \Longleftarrow{ }_{\mathrm{E}}^{*} M^{\prime}$.

Proof.

(i) By induction on the length of the reduction sequence $M \longrightarrow{ }_{\mathrm{R}}^{*} M^{\prime}$, using Lemma 4.7 .

(ii) By induction on the length of the reduction sequence $M \Longrightarrow{ }_{\mathrm{E}}^{*} N$, using Part (i).

Now, by Proposition 4.4(i), $\longrightarrow_{\mathrm{E}}^{*}=\Longrightarrow_{\mathrm{E}}^{*}$. Therefore Lemma 4.8(ii) implies that the relations $\longrightarrow_{\mathrm{E}}$ and $\longrightarrow_{\mathrm{R}}$ commute:

Proposition 4.9. If $N \longleftarrow_{\mathrm{E}}^{*} M \longrightarrow{ }_{\mathrm{R}}^{*} M^{\prime}$, then there is a $P$ such that $N \longrightarrow_{\mathrm{R}}^{*} P \longleftarrow{ }_{\mathrm{E}}^{*} M^{\prime}$.

4.3. Confluence of $\longrightarrow \mathbf{F P}$. We now use the results of Sections 4.1 and 4.2 to prove the main result of Section 4

Proposition 4.10. The relation $\longrightarrow \mathrm{FP}$ is confluent.

Proof. Proposition 4.3 states that $\longrightarrow_{\mathrm{R}}$ is confluent, Proposition 4.4(ii) states that $\longrightarrow_{\mathrm{E}}$ is confluent, and Proposition 4.9 states that $\longrightarrow_{\mathrm{R}}$ commutes with $\longrightarrow_{\mathrm{E}}$. By the HindleyRosen Lemma [2, p. 64], the relation $\longrightarrow_{\mathrm{FP}}=\longrightarrow_{\mathrm{R}} \cup \longrightarrow_{\mathrm{E}}$ is confluent.

Corollary 4.11 (Church-Rosser property). If $M=\mathrm{FP} N$, then there is a $P$ such that $M \longrightarrow{ }_{\mathrm{FP}}^{*} P$ and $N \longrightarrow{ }_{\mathrm{FP}}^{*} P$.

Proof. Follows from confluence of $\longrightarrow$ FP [2, p. 54].

\section{Remarks.}

(i) Orienting the axioms $(\overline{\mathrm{SP}})$ and $(\bar{\eta})$ of $\longrightarrow$ FP as contraction axioms does not give rise to a confluent reduction relation: with these axioms we would have the reductions $\lambda x . x \longleftarrow_{\mathrm{FP}}\left\langle\pi_{1}(\lambda x . x), \pi_{2}(\lambda x . x)\right\rangle \longrightarrow_{\mathrm{FP}}^{*}\left\langle\lambda x . \pi_{1} x, \lambda x . \pi_{2} x\right\rangle$, but the two terms $\lambda x . x$ and $\left\langle\lambda x . \pi_{1} x, \lambda x . \pi_{2} x\right\rangle$ would be normal forms.

(ii) The commutation axioms of $\lambda_{\mathrm{FP}}$ depend on the fact that the calculus is untyped, such that, intuitively, every function is also a pair and vice versa. A different line of work concerns reduction relations in typed calculi, with product and unit types, containing (SP) oriented as a contraction axiom [4].

\section{MAIN RESUlT}

We are now almost in a position to prove the main result: Suppose $M$ and $N$ are pure $\lambda$-terms such that $M={ }_{\beta \eta \mathrm{SP}} N$. Then $M={ }_{\mathrm{FP}} N$, and by the Church-Rosser property (Corollary 4.11) there is a $P$ such that $M \longrightarrow_{\mathrm{FP}}^{*} P$ and $N \longrightarrow{ }_{\mathrm{FP}}^{*} P$. However, since $\longrightarrow$ FP 
contains SP-expansion, we cannot immediately conclude that $P$ is a pure $\lambda$-term with $M \longrightarrow{ }_{\beta \eta}^{*} P$ and $N \longrightarrow{ }_{\beta \eta}^{*} P$.

Definition 5.1. The $\pi$-erasure of a $\lambda$-term $M$ is the pure $\lambda$-term $|M|$ defined inductively as follows:

$$
\begin{aligned}
|x| & =x \\
|M N| & =|M||N| \\
|\lambda x . M| & =\lambda x \cdot|M| \\
|\langle M, N\rangle| & =|M| \\
\left|\pi_{1} M\right| & =|M| \\
\left|\pi_{2} M\right| & =|M|
\end{aligned}
$$

We could just as well have defined $|\langle M, N\rangle|$ as $|N|$, since we are only interested in $|P|$ when $P$ is $\pi$-symmetric:

Definition 5.2. A $\lambda$-term $M$ is $\pi$-symmetric if for every subterm of $M$ of the form $\langle P, Q\rangle$, the $\pi$-erasures of $P$ and $Q$ are $\beta \eta$-equivalent: $|P|={ }_{\beta \eta}|Q|$.

In particular, every pure $\lambda$-term is $\pi$-symmetric.

\section{Proposition 5.3.}

(i) $|M[x:=N]|=|M|[x:=|N|]$

(ii) If $M$ and $N$ are $\pi$-symmetric, then $M[x:=N]$ is $\pi$-symmetric.

Proof. By induction on $M$.

Proposition 5.4. If $M$ is $\pi$-symmetric and $M \longrightarrow \mathrm{FP} N$, then

(i) $|M|={ }_{\beta \eta}|N|$, and

(ii) $N$ is $\pi$-symmetric.

Proof. By induction on the definition of $M \longrightarrow_{\mathrm{FP}} N$, using Proposition 5.3 ,

Now we are ready to prove that $\lambda_{\mathrm{FP}}$ is a conservative extension of $\lambda_{\beta \eta}$ :

Theorem 5.5. Let $M, N$ be pure $\lambda$-terms. If $M={ }_{\mathrm{FP}} N$, then $M={ }_{\beta \eta} N$.

Proof. Suppose $M$ and $N$ are pure $\lambda$-terms such that $M={ }_{\mathrm{FP}} N$. By the Church-Rosser property (Corollary 4.11) there is a $P$ such that $M \longrightarrow{ }_{\mathrm{FP}}^{*} P$ and $N \longrightarrow{ }_{\mathrm{FP}}^{*} P$. Since $M$ and $N$ are pure, they are in particular $\pi$-symmetric; it follows from Proposition 5.4 that $P$ is $\pi$-symmetric and that $|M|={ }_{\beta \eta}|P|={ }_{\beta \eta}|N|$. Hence $M=|M|={ }_{\beta \eta}|N|=N$.

Corollary 5.6. The theory $\lambda_{\mathrm{FP}}$ is consistent.

Proof. By Theorem [5.5 and consistency of $\lambda_{\beta \eta}$ [2, p. 67].

Finally we turn to the main result of this article:

Theorem 5.7. Let $M, N$ be pure $\lambda$-terms. If $M={ }_{\beta \eta \mathrm{SP}} N$, then $M={ }_{\beta \eta} N$.

Proof. By Theorem 5.5 and the fact that $\lambda_{\mathrm{FP}}$ is an extension of $\lambda_{\beta \eta \mathrm{SP}}$. 
We have also obtained a new - syntactic - proof of consistency of $\lambda_{\beta \eta \mathrm{SP}}$ :

Corollary 5.8. The theory $\lambda_{\beta \eta \mathrm{SP}}$ is consistent.

Remark. The question of conservativity was originally formulated in a slightly different setting [10]: let $D, D_{1}$ and $D_{2}$ be three new constants, and add the following axioms to the pure $\lambda_{\beta \eta}$-calculus:

$$
\begin{array}{rll}
D_{1}(D M N) & ={ }_{\beta \eta D} & M \\
D_{2}(D M N) & =\beta \eta D & N \\
D\left(D_{1} M\right)\left(D_{2} M\right) & ={ }_{\beta \eta D} & M
\end{array}
$$

To see that the resulting theory $\lambda_{\beta \eta D}$ is conservative over $\lambda_{\beta \eta}$, one can simulate $\lambda_{\beta \eta D}$ in $\lambda_{\beta \eta \mathrm{SP}}$ by defining $D$ as $\lambda x . \lambda y .\langle x, y\rangle, D_{1}$ as $\lambda x . \pi_{1} x$, and $D_{2}$ as $\lambda x . \pi_{2} x$.

\section{RELATED PROBLEMS}

The conservativity proof presented here can be adapted to the non-extensional case settled by de Vrijer [23], i.e., a minor modification gives an alternative proof that $\lambda_{\beta S P}$ is conservative over the lambda calculus $\lambda_{\beta}$. To this end, one should simply remove the axiom $(\eta)$ from every definition and proof. The electronic, formalized version of the proof allows for a straightforward verification that the modification is correct.

Another related problem posed by Klop and de Vrijer is still open: whether the reduction relation $\longrightarrow \beta \eta \mathrm{SP}$ has the unique normal-form property [10]. The theory $\lambda_{\mathrm{FP}}$ does not seem useful in solving that problem.

Meyer asked whether any lambda theory can be conservatively extended with surjective pairing [6]. That problem also remains open.

\section{ACKNOWLEDGEMENTS}

The author is grateful to Olivier Danvy, Andrzej Filinski, and the anonymous referees for their insightful comments. Vincent van Oostrom pointed out a substantial simplification of the confluence proof in Section 4. Thanks are also due to Pierre-Louis Curien and Soren Lassen for discussions on this work, and to Karl Crary for his lectures on LF and the Twelf system in the fall of 2004 at CMU.

The work described in this article is supported by BRICS (Basic Research in Computer Science (http://www.brics.dk), funded by the Danish National Research Foundation).

\section{REFERENCES}

[1] Henk Barendregt. Pairing without conventional restraints. Z. Math. Logik Grundlag. Math., 20:289-306, 1974.

[2] Henk Barendregt. The Lambda Calculus: Its Syntax and Semantics, volume 103 of Studies in Logic and the Foundation of Mathematics. North-Holland, revised edition, 1984.

[3] Henk Barendregt. Lambda calculi with types. In Samson Abramsky, Dov M. Gabbay, and Thomas S. E. Maibaum, editors, Handbook of Logic in Computer Science, Vol. 2, chapter 2, pages 118-309. Oxford University Press, Oxford, 1992.

[4] Pierre-Louis Curien and Roberto Di Cosmo. A confluent reduction system for the lambda-calculus with surjective pairing and terminal object. Journal of Functional Programming, 6(2):299-327, 1996.

[5] Pierre-Louis Curien and Thérèse Hardin. Yet yet a counterexample for $\lambda+$ SP. Journal of Functional Programming, 4(1):113-115, 1994.

[6] Nachum Dershowitz, Jean-Pierre Jouannaud, and Jan Willem Klop. Open problems in rewriting. In Ronald V. Book, editor, Rewriting Techniques and Applications, 4th International Conference, RTA91, volume 488 of Lecture Notes in Computer Science, pages 445-456. Springer-Verlag, 1991. The RTA list of open problems is currently maintained at http://www.lsv.ens-cachan.fr/rtaloop/. 
[7] Glenn Durfee. A model for a list-oriented extension of the lambda calculus. Master's thesis, School of Computer Science, Carnegie Mellon University, 1997.

[8] C. Barry Jay and Neil Ghani. The virtues of eta-expansion. Journal of Functional Programming, 5(2):135-154, 1995.

[9] Jan Willem Klop. Combinatory Reduction Systems. Mathematical Centre Tracts 127. Mathematisch Centrum, Amsterdam, 1980.

[10] Jan Willem Klop and Roel de Vrijer. Unique normal forms for lambda calculus with surjective pairing. Information and Computation, 80(2):97-113, 1989.

[11] Joachim Lambek and Philip J. Scott. Introduction to Higher Order Categorical Logic, volume 7 of Cambridge studies in advanced mathematics. Cambridge University Press, 1986.

[12] Soren B. Lassen. Head normal form bisimulation for pairs and the $\lambda \mu$-calculus. Manuscript, 2006.

[13] Tobias Nipkow. Orthogonal higher-order rewrite systems are confluent. In Marc Bezem and Jan Friso Groote, editors, Typed Lambda Calculi and Applications, TLCA '93, volume 664 of Lecture Notes in Computer Science, pages 306-317. Springer-Verlag, 1993.

[14] Vincent van Oostrom. Developing developments. Theoretical Computer Science, 175(1):159-181, 1997.

[15] Frank Pfenning. A proof of the Church-Rosser theorem and its representation in a logical framework. Technical Report CMU-CS-92-186, School of Computer Science, Carnegie Mellon University, 1992.

[16] Frank Pfenning and Carsten Schürmann. System description: Twelf - a meta-logical framework for deductive systems. In Harald Ganzinger, editor, Automated Deduction-CADE-16, 16th International Conference on Automated Deduction, volume 1632 of Lecture Notes in Computer Science, pages 202206. Springer-Verlag, 1999.

[17] Femke van Raamsdonk. Confluence and Normalization for higher-order rewriting. PhD thesis, Vrije Universiteit Amsterdam, 1996.

[18] György E. Révész. A list-oriented extension of the lambda-calculus satisfying the Church-Rosser theorem. Theoretical Computer Science, 93:75-89, 1992.

[19] György E. Révész. Categorical combinators with explicit products. Fundamenta Informaticae, 22:153166, 1995.

[20] Dana S. Scott. Logic and programming languages. Communications of the ACM, 20:634-641, 1977.

[21] Dana S. Scott. Relating theories of the lambda calculus. In J. P. Seldin and J. R. Hindley, editors, To H.B. Curry: Essays on Combinatory Logic, Lambda-Calculus and Formalism, pages 403-450. Academic Press, 1980.

[22] Masako Takahashi. Parallel reductions in $\lambda$-calculus. Information and Computation, 118:120-127, 1995.

[23] Roel de Vrijer. Extending the lambda calculus with surjective pairing is conservative. In Proceedings of the Fourth Annual IEEE Symposium on Logic in Computer Science, pages 204-215, Pacific Grove, California, June 1989. IEEE Computer Society Press.

\section{Appendix A. Formalized statement of the main Result}

Below is the formalized statement of the conservativity theorem. The full formal proof consists of 2670 lines of Twelf code. It was developed using version 1.5R1 of the Twelf system. ${ }^{5}$ The encoding technique is based on a formal proof of the Church-Rosser theorem for $\beta$-reduction that is distributed along with earlier versions of the Twelf system [15.

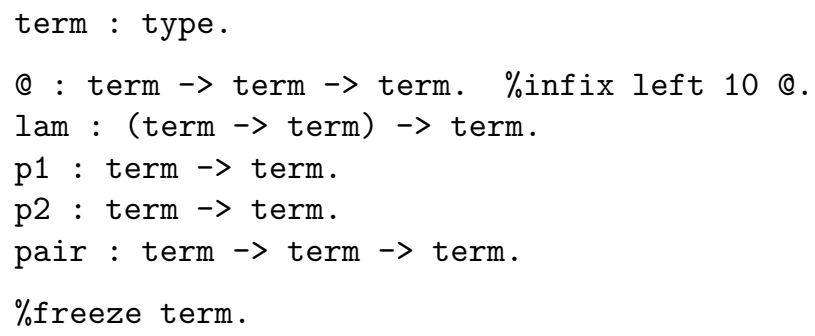

${ }^{5}$ The Twelf system can be obtained from http://www.cs.cmu.edu/ twelf/ 
$\% \%$ Lambda calculus with the extensionality rules eta and SP.

$==\mathrm{SP}:$ term $\rightarrow$ term $\rightarrow$ type. \%infix none $5==\mathrm{SP}$.

sp_beta : (lam F) @ $N==S P F N$.

sp_eta : $\operatorname{lam}([\mathrm{x}] \mathrm{M} @ \mathrm{x})==\mathrm{SP} \mathrm{M}$.

sp_proj1 : p1 (pair M N) ==SP M.

sp_proj2 : p2 (pair M N) ==SP N.

sp_SP : pair (p1 M) (p2 M) ==SP M.

$\%$ Congruence rules.

sp_refl : M ==SP $M$.

sp_sym : M ==SP $N \rightarrow N==S P M$.

sp_trans : $M==S P N \rightarrow N==S P P \rightarrow M==S P$.

sp_c-app : $M$ @ $N==S P M$ ' @ N'

$<-\mathrm{M}==\mathrm{SP} M$,

$<-\mathrm{N}==\mathrm{SP} \mathrm{N}^{\prime}$.

sp_c-lam : lam $F==S P$ lam $F^{\prime}$

$<-\left(\{\mathrm{x}\} \mathrm{F} \mathrm{x}==\mathrm{SP} \mathrm{F}^{\prime} \mathrm{x}\right)$.

sp_c-p1 : p1 M ==SP p1 M'

$<-M==S P M^{\prime}$.

sp_c-p2 : p2 M ==SP p2 M'

$<-\mathrm{M}==\mathrm{SP} M$, .

sp_c-pair : pair $\mathrm{M} N==\mathrm{SP}$ pair $\mathrm{M}^{\prime} \mathrm{N}^{\prime}$

$<-\mathrm{M}==\mathrm{SP} M$,

$<-N==S P N^{\prime}$.

$\%$ freeze $==$ SP.

$\% \%$ Pure lambda-terms, i.e., no "pair", "p1", or "p2".

pterm : type.

- : pterm $\rightarrow$ pterm $\rightarrow$ pterm. \%infix left 10 .

lambda : (pterm $\rightarrow$ pterm) $\rightarrow$ pterm.

$\%$ freeze pterm.

$\% \%$ Beta-eta equality on pure terms.

$==$ be : pterm $\rightarrow$ pterm $\rightarrow$ type. \%infix none $5==$ be.

be_beta : (lambda F) - $\mathrm{N}==$ be $F \mathrm{~N}$.

be_eta : lambda $([\mathrm{x}] \mathrm{M} \sim \mathrm{x})==$ be $\mathrm{M}$.

$\%$ Congruence rules.

be_refl : $M==b e ~ M$.

be_sym : $M==$ be $N \rightarrow N==$ be $M$. 


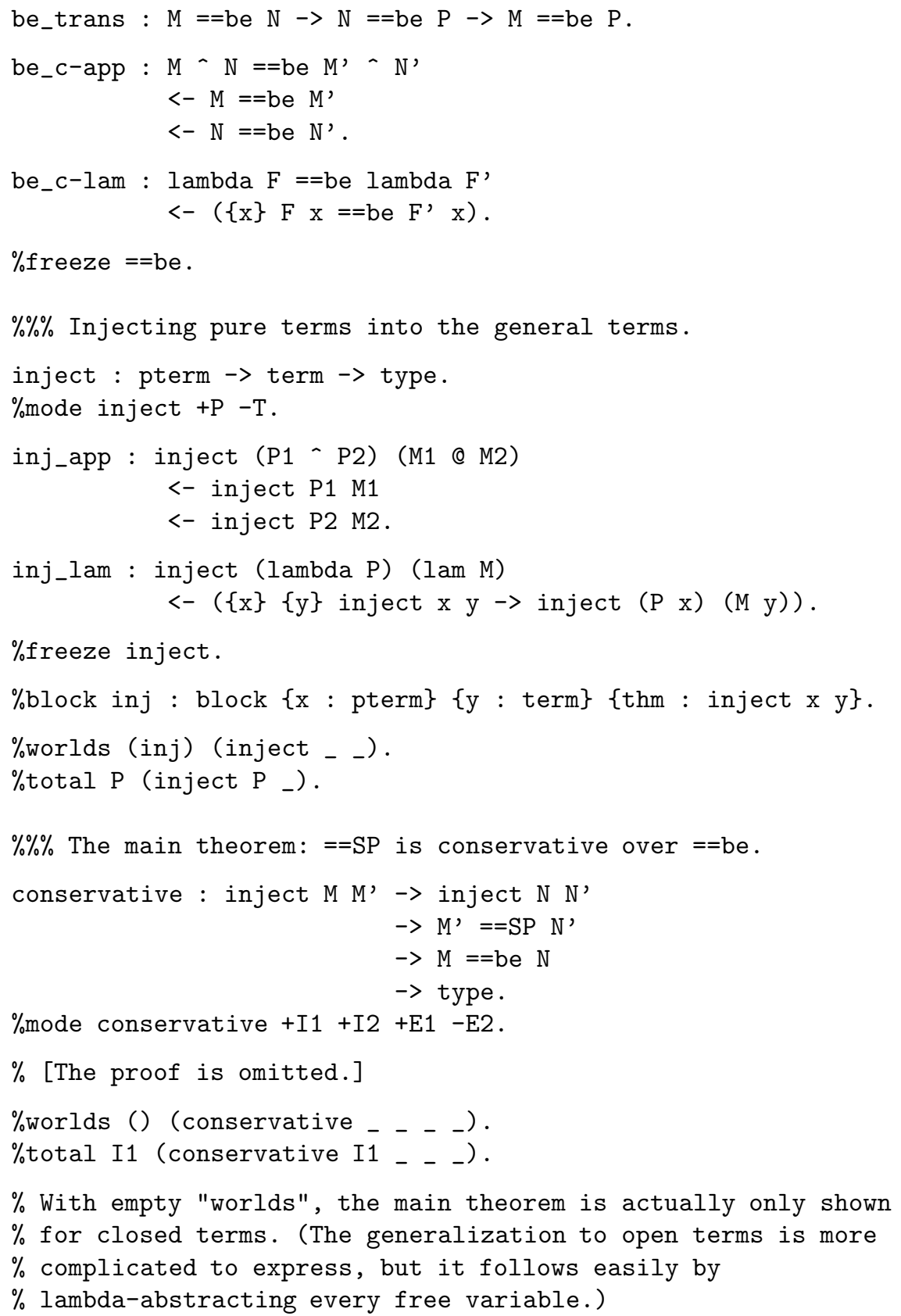

